\title{
Oscillation Criteria for Some Higher Order Integrodynamic Equations on Timescales
}

\author{
Said R. Grace ${ }^{1}$ and Mohamed A. El-Beltagy ${ }^{1,2}$ \\ ${ }^{1}$ Engineering Mathematics Department, Engineering Faculty, Cairo University, Giza 12221, Egypt \\ ${ }^{2}$ Electrical and Computer Engineering Department, Engineering Faculty, Effat University, Jeddah 21478, Saudi Arabia \\ Correspondence should be addressed to Mohamed A. El-Beltagy; zbeltagy@hotmail.com
}

Received 23 June 2015; Accepted 25 August 2015

Academic Editor: Allan Peterson

Copyright (c) 2015 S. R. Grace and M. A. El-Beltagy. This is an open access article distributed under the Creative Commons Attribution License, which permits unrestricted use, distribution, and reproduction in any medium, provided the original work is properly cited.

\begin{abstract}
We study the oscillation behavior for some higher order integrodynamic equations on timescales. We establish some new sufficient conditions guaranteeing that all solutions of theses equations are oscillatory. Some numerical examples in the continuous case are given to validate the theoretical results.
\end{abstract}

\section{Introduction}

Integrodynamic equations on timescales are an important topic with applications in many physical systems. For general basic ideas and background, we refer to [1]. Oscillation results of integral equations of Volterra type are scant and only few results exist on this subject. Related studies can be found in [2-6]. In this paper, we investigate the oscillatory behavior of the solutions of some higher order integrodynamic equations on timescale in the form

$$
\left(r x^{\Delta}\right)^{\Delta^{n-1}}(t)=e(t)-\int_{0}^{t} a(t, s) F(s, x(s)) \Delta s .
$$

To the best of our knowledge, there appear to be no such results on the oscillation of (1). Therefore, our main goal here is to initiate such a study by establishing some new criteria for the oscillation of (1) and other related equations. This work is an extension to the analysis done in [7]. The nonoscillatory behavior for some higher order integrodynamic equations was studied recently in [8].

We take $T \subseteq R$ to be an arbitrary timescale with $0 \in T$ and $\operatorname{Sup} T=\infty$.

Whenever we write $t \geq s$, we mean $t \in[s, \infty) \cap T$.

We assume throughout the following:

(I) $e, r: T \rightarrow R$ and $a: T \times T \rightarrow R$ are rd-continuous functions, $r(t)>0$, and $a(t, s) \geq 0$ for $t \geq s$; and there exist rd-continuous functions $p, q: T \rightarrow R^{+}$such that

$$
\begin{aligned}
a(t, s) & \leq p(t) q(s) \quad \text { for } t \geq s, \\
\sup p(t) & :=k_{1}<\infty, \\
\sup _{t \geq 0} \int_{0}^{t} q(s) \Delta s & :=k_{2}<\infty ;
\end{aligned}
$$

(II) $F: T \times R \rightarrow R$ is continuous and assume that there exist $f_{1}, f_{2}: T \times R \rightarrow R$ continuous functions such that $F(t, x)=f_{1}(t, x)-f_{2}(t, x)$ for $t \geq 0$ and $x f_{i}(t, x)>0$ for $x \neq 0, t \geq 0$, and $i=1,2$;

(III) there exist constants $\beta$ and $\gamma$ which are the ratios of positive odd integers and $p_{i}: T \rightarrow R^{+}=(0, \infty)$, $i=1,2$, such that

$$
\begin{aligned}
& f_{1}(t, x) \geq p_{1}(t) x^{\beta}, \\
& f_{2}(t, x) \leq p_{2}(t) x^{\gamma},
\end{aligned}
$$$$
\text { for } x>0, t \geq 0 \text {, }
$$

$$
\begin{aligned}
& f_{1}(t, x) \leq p_{1}(t) x^{\beta}, \\
& f_{2}(t, x) \geq p_{2}(t) x^{\gamma},
\end{aligned}
$$

$$
\text { for } x<0, t \geq 0 \text {. }
$$


By a solution of (1) we mean a $\Delta$-differential function defined on $T$ that is nontrivial in every neighborhood of infinity. A solution $x(t)$ of (1) is said to be oscillatory if there exists $\left\{t_{n}\right\} \subseteq$ $T$ such that $x\left(t_{n}\right)=0$ or $x(t) x(\sigma(t))<0$; otherwise, it is called nonoscillatory.

\section{Auxiliary Results}

We employ the following lemmas.

Lemma 1 (see [9]). If $X$ and $Y$ are nonnegative, then

$$
\begin{array}{ll}
X^{\lambda}+(\lambda-1) Y^{\lambda}-\lambda X Y^{\lambda-1} \geq 0 ; & \lambda>1, \\
X^{\lambda}-(1-\lambda) Y^{\lambda}-\lambda X Y^{\lambda-1} \leq 0 ; & \lambda<1,
\end{array}
$$

where equality holds if and only if $X=Y$.

Lemma 2 (Young's inequality). Let $X, Y \geq 0, n>1$, and $1 / n+$ $1 / m=1$, then

$$
X Y \leq \frac{1}{n} X^{n}+\frac{1}{m} Y^{m}
$$

and equality holds if and only if $Y=X^{n-1}$.

Lemma 3 (see [3, corollary 1]). Assume that $n \in N, s, t \in T$, and $f: T \rightarrow R$ is rd-continuous function, and then

$$
\begin{aligned}
& \int_{s}^{t} \int_{\eta_{n}}^{t} \cdots \int_{\eta_{2}}^{t} f\left(\eta_{1}\right) \Delta \eta_{1} \Delta \eta_{2} \cdots \Delta \eta_{n} \\
& \quad=(-1)^{n-1} \int_{s}^{t} h_{n-1}(s, \sigma(\eta)) f(\eta) \Delta \eta .
\end{aligned}
$$

In Lemma $3, h_{n}$ stand for the Taylor monomials (see [1, section 1.6]) which are defined recursively by

$$
\begin{aligned}
h_{0}(t, s) & =1, \\
h_{n+1}(t, s) & =\int_{s}^{t} h_{n}(\tau, s) \Delta \tau \\
\text { for } t, s & \in T, n \in N .
\end{aligned}
$$

It follows that $h_{1}(t, s)=t-s$ for any timescale, but simple formulas, in general, do not hold for $n \geq 2$.

For $t \geq s \in T$, we define

$$
\begin{gathered}
H_{n-1}(t, s) \geq h_{0}(t, s)+h_{1}(t, s)+\cdots+h_{n-1}(t, s), \\
H\left(t, t_{0}\right)=\int_{t_{0}}^{t} \frac{1}{r(s)} H_{n-1}\left(s, t_{0}\right) \Delta s \quad \forall t_{0} \geq 0 .
\end{gathered}
$$

\section{Main Results}

In this section we present the following main results.

Theorem 4. Let conditions (I) and (II) hold with $f_{2}=0$. If

$$
\begin{aligned}
& \limsup _{t \rightarrow \infty} \frac{1}{H\left(t, t_{0}\right)} \int_{t_{0}}^{t} \frac{1}{r(u)} \int_{t_{0}}^{u} h_{n-2}(u, s) e(s) \Delta s \Delta u \\
& \quad=\infty \\
& \liminf _{t \rightarrow \infty} \frac{1}{H\left(t, t_{0}\right)} \int_{t_{0}}^{t} \frac{1}{r(u)} \int_{t_{0}}^{u} h_{n-2}(u, s) e(s) \Delta s \Delta u \\
& \quad=-\infty,
\end{aligned}
$$

for all $t_{0} \geq 0$, then (1) is oscillatory.

Proof. Let $x(t)$ be a nonoscillatory solution of (1). Hence either $x(t)$ is eventually positive or $x(t)$ is eventually negative.

First assume $x(t)$ is eventually positive. Fix $t_{0} \geq 0$ and suppose $x(t)>0$ for $t \geq t_{1}$ for some $t_{1} \geq t_{0}$. From (1), we see that

$$
\begin{aligned}
\left(r x^{\Delta}\right)^{\Delta^{n-1}}(t)= & e(t)-\int_{0}^{t_{1}} a(t, s) F(s, x(s)) \Delta s \\
& -\int_{t_{1}}^{t} a(t, s) f_{1}(s, x(s)) \Delta s \\
\leq & e(t)-\int_{0}^{t_{1}} a(t, s) F(s, x(s)) \Delta s
\end{aligned}
$$

Let

$$
b:=\max _{0 \leq t \leq t_{1}}|F(t, x(t))|<\infty .
$$

By assumption (3), we have

$$
\begin{aligned}
& \left|-\int_{0}^{t_{1}} a(t, s) F(s, x(s)) \Delta s\right| \\
& \quad \leq \int_{0}^{t_{1}} a(t, s)|F(s, x(s))| \Delta s \leq b \int_{0}^{t_{1}} p(t) q(s) \Delta s \\
& \quad \leq b k_{1} \int_{0}^{t_{1}} q(s) \Delta s \leq b k_{1} k_{2}:=k .
\end{aligned}
$$

Hence, from (12), we get

$$
\left(r x^{\Delta}\right)^{\Delta^{n-1}}(t) \leq e(t)+k, \quad \forall t \geq t_{1}
$$


Integrating (15) $(n-1)$-times from $t_{1}$ to $t$ and then using Lemma 3, we obtain

$$
\begin{aligned}
\left(r x^{\Delta}\right)(t) \leq & h_{0}\left(t, t_{1}\right)\left(r x^{\Delta}\right)\left(t_{1}\right) \\
& +h_{1}\left(t, t_{1}\right)\left(r x^{\Delta}\right)^{\Delta}\left(t_{1}\right)+\cdots \\
& +h_{n-2}\left(t, t_{1}\right)\left(r x^{\Delta}\right)^{\Delta^{n-2}}\left(t_{1}\right)+k h_{n-1}\left(t, t_{1}\right) \\
& +\int_{t_{1}}^{t} \int_{t_{1}}^{\xi_{n-1}} \cdots \int_{t_{1}}^{\xi_{2}} e\left(\xi_{1}\right) \Delta \xi_{1} \Delta \xi_{2} \cdots \Delta \xi_{n-1} \\
= & k h_{n-1}\left(t, t_{1}\right)+\sum_{i=0}^{n-2} h_{i}\left(t, t_{1}\right)\left(r x^{\Delta}\right)^{\Delta^{i}}\left(t_{1}\right) \\
& +\int_{t_{1}}^{t} h_{n-2}(t, \sigma(s)) e(s) \Delta s .
\end{aligned}
$$

From the properties of the functions $h_{i}$ and the definition of the function $H_{n-1}\left(t, t_{1}\right)$ for all $t_{1} \geq 0$, we get

$$
\left(r x^{\Delta}\right)(t) \leq c H_{n-1}\left(t, t_{1}\right)+\int_{t_{1}}^{t} h_{n-2}(t, \sigma(s)) e(s) \Delta s,
$$

where

$$
c:=\max \left\{k, \max _{0 \leq i \leq n-2}\left|\left(r x^{\Delta}\right)^{\Delta^{i}}\left(t_{1}\right)\right|\right\} .
$$

Dividing (17) by $r(t)$ and hence integrating from $t_{1}$ to $t$ we obtain

$$
\begin{aligned}
x(t) \leq & x\left(t_{1}\right)+c H\left(t, t_{1}\right) \\
& +\int_{t_{1}}^{t} \frac{1}{r(u)} \int_{t_{1}}^{u} h_{n-2}(u, \sigma(s)) e(s) \Delta s \Delta u \\
\leq & x\left(t_{1}\right)+c H\left(t, t_{0}\right) \\
& +\int_{t_{0}}^{t} \frac{1}{r(u)} \int_{t_{0}}^{u} h_{n-2}(u, \sigma(s)) e(s) \Delta s \Delta u .
\end{aligned}
$$

Dividing (19) by $H\left(t, t_{0}\right)$ and taking lim inf of both sides of (19) as $t \rightarrow \infty$, we obtain a contradiction to the fact that $x(t)>0$ for $t \geq t_{1}$. The proof of the case when $x(t)$ is eventually negative is similar. This completes the proof.

From the proof of Theorem 4, one can easily extract the following result on the asymptotic behavior of the nonoscillatory solutions of (1).

Theorem 5. Let conditions (I) and (II) hold with $f_{2}=0$ and suppose

$$
\begin{aligned}
& \limsup _{t \rightarrow \infty} \frac{1}{H\left(t, t_{0}\right)} \int_{t_{0}}^{t} \frac{1}{r(u)} \int_{t_{0}}^{u} e(s) \Delta s \Delta u<\infty, \\
& \liminf _{t \rightarrow \infty} \frac{1}{H\left(t, t_{0}\right)} \int_{t_{0}}^{t} \frac{1}{r(u)} \int_{t_{0}}^{u} e(s) \Delta s \Delta u>-\infty
\end{aligned}
$$

for all $t_{0} \geq 0$. If $x(t)$ is nonoscillatory solution of (1), then

$$
x(t)=O\left(H\left(t, t_{0}\right)\right) \text { as } t \longrightarrow \infty .
$$

Next, we present the following result.
Theorem 6. Let conditions (I) and (II) hold with $f_{1}=0$ and $f_{2}$ is nondecreasing in the second variable. If conditions (11) hold for every constant $c>0$,

$$
\begin{array}{r}
\lim _{t \rightarrow \infty} \frac{1}{H\left(t, t_{0}\right)} \int_{t_{0}}^{t} \frac{1}{r(u)} \int_{t_{0}}^{u} h_{n-2}(u, \sigma(\tau)) \\
\cdot \int_{t_{0}}^{\tau} a(\tau, s)\left|f_{2}(s, c)\right| \Delta s \Delta \tau \Delta u<\infty,
\end{array}
$$

for any $t_{0} \geq 0$, then every bounded solution of (1) is oscillatory.

Proof. Let $x(t)$ be a bounded nonoscillatory solution of (1) and assume that $x(t)$ is eventually positive. Fix $t_{0} \geq 0$ and suppose $x(t)>0$ for $t \geq t_{1}$ for some $t_{1} \geq t_{0}$ and $x(t) \leq c_{1}$ for some constant $c_{1}>0$. From (1), we have

$$
\begin{aligned}
\left(r x^{\Delta}\right)^{\Delta^{n-1}}(t)= & e(t)-\int_{0}^{t_{1}} a(t, s) F(s, x(s)) \Delta s \\
& +\int_{t_{1}}^{t} a(t, s) f_{2}(s, x(s)) \Delta s .
\end{aligned}
$$

Proceeding as in the proof of Theorem 4, we get (14). Thus,

$$
\left(r x^{\Delta}\right)^{\Delta^{n-1}}(t) \leq k+e(t)+\int_{t_{1}}^{t} a(t, s) f_{2}\left(s, c_{1}\right) \Delta s
$$

The rest of the proof is similar to that of Theorem 4 and hence it is omitted.

Theorem 7. Let conditions (I) and (II) hold with $\beta>1$ and $\gamma=1$ and suppose that conditions (11) hold and

$$
\begin{aligned}
& \lim _{t \rightarrow \infty} \frac{1}{H\left(t, t_{0}\right)} \int_{t_{0}}^{t} \frac{1}{r(u)} \int_{t_{0}}^{u} h_{n-2}(u, \sigma(\tau)) \\
& \quad \cdot \int_{t_{0}}^{\tau} a(\tau, s) p_{1}^{1 /(\beta-1)}(s) p_{2}^{\beta /(\beta-1)}(s) \Delta s \Delta \tau \Delta u<\infty
\end{aligned}
$$

for all $t_{0} \geq 0$. Then (1) is oscillatory.

Proof. Let $x(t)$ be a nonoscillatory solution of (1), Hence either $x(t)$ is eventually positive or $x(t)$ is eventually negative. First, assume $x(t)$ is eventually positive. Fix $t_{0} \geq 0$ and assume $x(t)>0$ for $t \geq t_{1}$ for some $t_{1} \geq t_{0}$.

Using conditions (I) and (II) with $\beta>1$ and $\gamma=1$ in (1), we have

$$
\begin{aligned}
& \left(r x^{\Delta}\right)^{\Delta^{n-1}}(t) \\
& \leq e(t)-\int_{0}^{t_{1}} a(t, s) F(s, x(s)) \Delta s \\
& \quad+\int_{t_{1}}^{t} a(t, s)\left[p_{2}(s) x(s)-p_{1}(s) x^{\beta}(s)\right] \Delta s
\end{aligned}
$$


for $t \geq t_{1}$. Proceeding as in the proof of Theorem 4, we get (14) and hence

$$
\begin{aligned}
& \left(r x^{\Delta}\right)^{\Delta^{n-1}}(t) \\
& \leq k+e(t) \\
& \quad+\int_{t_{1}}^{t} a(t, s)\left[p_{2}(s) x(s)-p_{1}(s) x^{\beta}(s)\right] \Delta s
\end{aligned}
$$

for $t \geq t_{1}$.

By applying (5) with

$$
\begin{aligned}
\lambda & =\beta, \\
X & =p_{1}^{1 / \beta}(t) x(t), \\
Y & =\left(\frac{1}{\beta} p_{2}(t) p_{1}^{-1 / \beta}(t)\right)^{1 /(\beta-1)},
\end{aligned}
$$

we obtain

$$
\begin{aligned}
& p_{2}(t) x(t)-p_{1}(t) x^{\beta}(t) \\
& \leq(\beta-1) \beta^{\beta /(1-\beta)} p_{1}^{1 /(1-\beta)}(t) p_{2}^{\beta /(1-\beta)}(t), \\
& \quad \text { for } t \geq t_{1} .
\end{aligned}
$$

Using (29) in (26), we find

$$
\left(r x^{\Delta}\right)^{\Delta^{n-1}}(t) \leq k+e(t)+G(t), \quad \text { for } t \geq t_{1},
$$

where

$$
\begin{aligned}
& G(t)=(\beta-1) \\
& \cdot \beta^{\beta /(1-\beta)} \int_{t_{1}}^{t} a(t, s) p_{1}^{1 /(1-\beta)}(s) p_{2}^{\beta /(1-\beta)}(s) \Delta s .
\end{aligned}
$$

Integrating (30) $n$-times from $t_{1}$ to $t$ and then using Lemma 3 , we have

$$
\begin{aligned}
x(t) \leq & x\left(t_{1}\right)+c H\left(t, t_{1}\right) \\
& +\int_{t_{1}}^{t} \frac{1}{r(u)} \int_{t_{1}}^{u} h_{n-2}(u, \sigma(s)) e(s) \Delta s \Delta u \\
& +\int_{t_{1}}^{t} \frac{1}{r(u)} \int_{t_{1}}^{u} h_{n-2}(u, \sigma(s)) G(s) \Delta s \Delta u \\
\leq & x\left(t_{1}\right)+c H\left(t, t_{0}\right) \\
& +\int_{t_{0}}^{t} \frac{1}{r(u)} \int_{t_{1}}^{u} h_{n-2}(u, \sigma(s)) e(s) \Delta s \Delta u \\
& +\int_{t_{0}}^{t} \frac{1}{r(u)} \int_{t_{0}}^{u} h_{n-2}(u, \sigma(s)) G(s) \Delta s \Delta u,
\end{aligned}
$$

where $c$ is given in (18). The rest of the proof is similar to that of the proof of Theorem 4 and hence is omitted.
Theorem 8. Let conditions (I) and (II) hold with $\beta=1$ and $\gamma<1$. If, in addition to conditions (11), we suppose

$$
\begin{aligned}
& \lim _{t \rightarrow \infty} \frac{1}{H\left(t, t_{0}\right)} \int_{t_{0}}^{t} \frac{1}{r(u)} \int_{t_{0}}^{u} h_{n-2}(u, \sigma(\tau)) \\
& \quad \cdot \int_{t_{0}}^{\tau} a(\tau, s) p_{1}^{\gamma /(\gamma-1)}(s) p_{2}^{1 /(\gamma-1)}(s) \Delta s \Delta \tau \Delta u<\infty,
\end{aligned}
$$

for any $t_{0} \geq 0$, then (1) is oscillatory.

Proof. Let $x(t)$ be a nonoscillatory solution of (1). First, assume $x(t)$ is eventually positive. Fix $t_{0} \geq 0$ and suppose $x(t)>0$ for $t \geq t_{1}$ for some $t_{1} \geq t_{0}$. Using conditions (I) and (II) with $\beta=1$ and $\gamma<1$ in (1), we find

$$
\begin{aligned}
\left(r x^{\Delta}\right)^{\Delta^{n-1}}(t) & \\
\leq & e(t)-\int_{0}^{t_{1}} a(t, s) F(s, x(s)) \Delta s \\
& \quad+\int_{t_{1}}^{t} a(t, s)\left[p_{2}(s) x^{\gamma}(s)-p_{1}(s) x(s)\right] \Delta s
\end{aligned}
$$

for $t \geq t_{1}$. Hence

$$
\begin{aligned}
& \left(r x^{\Delta}\right)^{\Delta^{n-1}}(t) \\
& \leq k+e(t) \\
& \quad+\int_{t_{1}}^{t} a(t, s)\left[p_{2}(s) x^{\gamma}(s)-p_{1}(s) x(s)\right] \Delta s
\end{aligned}
$$

for $t \geq t_{1}$,

where $k$ is defined as in the proof of Theorem 4. By applying (6) with

$$
\begin{aligned}
\lambda & =\gamma, \\
X & =p_{2}^{1 / \gamma}(t) x(t), \\
Y & =\left(\frac{1}{\gamma} p_{1}(t) p_{2}^{-1 / \gamma}(t)\right)^{1 /(\gamma-1)},
\end{aligned}
$$

we obtain

$$
\begin{aligned}
& p_{2}(t) x^{\gamma}(t)-p_{1}(t) x(t) \\
& \quad \leq(1-\gamma) \gamma^{\gamma /(\gamma-1)} p_{1}^{\gamma /(\gamma-1)}(t) p_{2}^{1 /(\gamma-1)}(t),
\end{aligned}
$$

for $t \geq t_{1}$.

Using (37) in (35), we find

$$
\begin{aligned}
& \left(r x^{\Delta}\right)^{\Delta^{n-1}}(t) \leq k+e(t)+(1-\gamma) \\
& \cdot \gamma^{\gamma /(1-\gamma)} \int_{t_{1}}^{t} a(t, s) p_{1}^{\gamma /(\gamma-1)}(s) p_{2}^{1 /(1-\gamma)}(s) \Delta s .
\end{aligned}
$$

The rest of the proof is similar to the proof of Theorem 4 and hence is omitted. 
Next, we present the following result with different nonlinearities, that is, with $\beta>1$ and $\gamma<1$.

Theorem 9. Let conditions (I) and (II) hold with $\beta>1$ and $\gamma<1$ and suppose that there exists a positive $r d$-continuous function $g: T \rightarrow R^{+}$such that

$$
\begin{gathered}
\lim _{t \rightarrow \infty} \frac{1}{H\left(t, t_{0}\right)} \int_{t_{0}}^{t} \frac{1}{r(u)} \int_{t_{0}}^{u} h_{n-2}(u, \sigma(\tau)) \int_{t_{0}}^{\tau} a(\tau, s) \\
\cdot\left[c_{1} g^{\beta /(\beta-1)}(s) p_{1}^{1 /(1-\beta)}(s)\right. \\
\left.+c_{2} g^{\gamma /(\gamma-1)}(s) p_{2}^{1 /(1-\gamma)}(s)\right] \Delta s \Delta \tau \Delta u<\infty
\end{gathered}
$$

for all $t_{0} \geq 0$, where

$$
\begin{aligned}
& c_{1}=(\beta-1) \beta^{\beta /(1-\beta)}, \\
& c_{2}=(1-\gamma) \gamma^{\gamma /(1-\gamma)} .
\end{aligned}
$$

If conditions (11) hold for all $t_{0} \geq 0$, then (1) is oscillatory.

Proof. Let $x(t)$ be a nonoscillatory solution of (1). First, assume $x(t)$ is eventually positive. Fix $t_{0} \geq 0$ and suppose $x(t)>0$ for $t \geq t_{1}$ for some $t_{1} \geq t_{0}$. Using conditions (I) and (II) in (1), we have

$$
\begin{aligned}
& \left(r x^{\Delta}\right)^{\Delta^{n-1}}(t) \\
& \leq e(t)-\int_{0}^{t_{1}} a(t, s) F(s, x(s)) \Delta s \\
& \quad+\int_{t_{1}}^{t} a(t, s)\left[g(s) x(s)-p_{1}(s) x^{\beta}(s)\right] \Delta s \\
& \quad+\int_{t_{1}}^{t} a(t, s)\left[p_{2}(s) x^{\gamma}(s)-g(s) x(s)\right] \Delta s
\end{aligned}
$$

for $t \geq t_{1}$.

As in the proof of Theorems 7 and 8, one can easily find

$$
\begin{aligned}
& \left(r x^{\Delta}\right)^{\Delta^{n-1}}(t) \leq k+e(t)+\int_{t_{1}}^{t} a(t, s) \\
& \cdot\left[(\beta-1) \beta^{\beta /(1-\beta)} g^{\beta /(\beta-1)}(s) p_{1}^{1 /(1-\beta)}(s)\right. \\
& \left.+(1-\gamma) \gamma^{\gamma /(1-\gamma)} g^{\gamma /(\gamma-1)}(s) p_{2}^{1 /(1-\gamma)}(s)\right] \Delta s .
\end{aligned}
$$

The rest of the proof is similar to that of Theorem 4 and hence is omitted.

For the cases when both functions $f_{1}$ and $f_{2}$ are superlinear, that is, $\beta>\gamma>1$, or sublinear, that is, $1>\beta>\gamma>0$, we present the following result.

Theorem 10. Let conditions (I) and (II) hold with $\beta>\gamma$. If, in addition to conditions (11), we suppose

$$
\begin{aligned}
& \lim _{t \rightarrow \infty} \frac{1}{H\left(t, t_{0}\right)} \int_{t_{0}}^{t} \frac{1}{r(u)} \int_{t_{0}}^{u} h_{n-2}(u, \sigma(\tau)) \\
& \quad \cdot \int_{t_{0}}^{\tau} a(\tau, s) p_{1}^{\gamma /(\gamma-\beta)}(s) p_{2}^{\beta /(\beta-\gamma)}(s) \Delta s \Delta \tau \Delta u<\infty,
\end{aligned}
$$

for all $t_{0} \geq 0$, then (1) is oscillatory.
Proof. Let $x(t)$ be a nonoscillatory solution of (1). First, assume $x(t)$ is eventually positive. Fix $t_{0} \geq 0$ and suppose $x(t)>0$ for $t \geq t_{1}$ for some $t_{1} \geq t_{0}$. Using conditions (I) and (II) in (1) with $\gamma<1$ and $\beta>1$, we have

$$
\begin{aligned}
& \left(r x^{\Delta}\right)^{\Delta^{n-1}}(t) \\
& \leq e(t)-\int_{0}^{t_{1}} a(t, s) F(s, x(s)) \Delta s \\
& \quad+\int_{t_{1}}^{t} a(t, s)\left[p_{2}(s) x^{\gamma}(s)-p_{1}(s) x^{\beta}(s)\right] \Delta s
\end{aligned}
$$

for $t \geq t_{1}$. By applying Lemma 2 with

$$
\begin{aligned}
& n=\frac{\beta}{\gamma}>1, \\
& X=x^{\gamma}(s), \\
& Y=\frac{\gamma}{\beta} \frac{p_{2}(s)}{p_{1}(s)}, \\
& m=\frac{\beta}{\beta-\gamma},
\end{aligned}
$$

we find

$$
\begin{aligned}
p_{2}(s) & x^{\gamma}(s)-p_{1}(s) x^{\beta}(s) \\
= & \frac{\beta}{\gamma} p_{1}(s)\left[x^{\gamma}(s)\left(\frac{\gamma}{\beta} \frac{p_{2}(s)}{p_{1}(s)}\right)-\frac{\gamma}{\beta}\left(x^{\gamma}(s)\right)^{\beta / \gamma}\right] \\
= & \frac{\beta}{\gamma} p_{1}(s)\left[X Y-\frac{1}{n} X^{n}\right] \leq \frac{\beta}{\gamma} p_{1}(s)\left(\frac{1}{m} Y^{m}\right) \\
= & \left(\frac{\beta-\gamma}{\gamma}\right)\left[\frac{\gamma}{\beta} p_{2}(s)\right]^{\beta /(\beta-\gamma)}\left(p_{1}(s)\right)^{\gamma /(\gamma-\beta)} .
\end{aligned}
$$

Using (46) in (44), we have

$$
\begin{gathered}
\left(r x^{\Delta}\right)^{\Delta^{n-1}}(t) \leq k+e(t)+\left(\frac{\beta-\gamma}{\gamma}\right)\left(\frac{\gamma}{\beta}\right)^{\beta /(\beta-\gamma)} \\
\cdot \int_{t_{1}}^{t} a(t, s) p_{2}^{\beta /(\beta-\gamma)}(s) p_{1}^{\gamma /(\gamma-\beta)}(s) \Delta s,
\end{gathered}
$$

for $t \geq t_{1}$. The rest of the proof is similar to the proof of Theorem 4 and hence is omitted.

Remark 11. The results of this section will remain the same if we replace condition (3) of assumption (I) by

$$
\sup _{t \geq t_{0}} \int_{0}^{t_{0}} a(t, s) \Delta s:=\bar{k}<\infty, \quad \forall t_{0} \geq 0,
$$

with $\bar{k}=k_{1} k_{2}$.

Remark 12. We note that we can obtain criteria on the asymptotic behavior of the nonoscillatory solutions of (1) similar to Theorem 5 . The details are left to the reader. 


\section{Further Oscillation Results}

This section is devoted to the study of the oscillatory properties of (1) with $f_{1}=0$.

Theorem 13. Let conditions (I) and (II) hold with $f_{1}=0$ and $\gamma<1$. Assume that there exists a rd-continuous function $p$ : $T \rightarrow R^{+}$such that

$$
\begin{aligned}
& \lim _{t \rightarrow \infty} \frac{1}{t} \int_{t_{0}}^{t} \frac{1}{r(u)} \int_{t_{0}}^{u} h_{n-2}(u, \sigma(\tau)) \\
& \quad \cdot \int_{t_{0}}^{\tau} p^{\gamma /(\gamma-1)}(s) p_{2}^{1 /(1-\gamma)}(s) q^{1 /(1-\gamma)}(s) \Delta s \Delta \tau \Delta u \\
& \quad<\infty, \\
& \frac{H_{n-1}\left(t, t_{0}\right)}{r(t)} \text { is bounded for } t \geq t_{0}, \\
& \lim _{t \rightarrow \infty} H\left(t, t_{0}\right)<\infty, \\
& \int^{\infty} s p(s) \Delta s<\infty .
\end{aligned}
$$

If

$$
\begin{aligned}
& \lim _{t \rightarrow \infty} \sup \frac{1}{t} \int_{t_{0}}^{t} \frac{1}{r(u)} \int_{t_{0}}^{u} h_{n-2}(u, \sigma(s)) e(s) \Delta s \Delta u \\
& \quad<\infty, \\
& \lim _{t \rightarrow \infty} \inf \frac{1}{t} \int_{t_{0}}^{t} \frac{1}{r(u)} \int_{t_{0}}^{u} h_{n-2}(u, \sigma(s)) e(s) \Delta s \Delta u \\
& >-\infty
\end{aligned}
$$

for all $t_{0} \geq 0$, then every nonoscillatory solution $x(t)$ of (1) satisfies

$$
\lim _{t \rightarrow \infty} \sup \frac{|x(t)|}{t}<\infty
$$

Proof. Let $x(t)$ be a nonoscillatory solution of (1). First, assume $x(t)$ is eventually positive. Fix $t_{0} \geq 0$ and suppose $x(t)>0$ for $t \geq t_{1}$ for some $t_{1} \geq t_{0}$. Using conditions (I) and (II) in (1), we have

$$
\begin{gathered}
\left(r x^{\Delta}\right)^{\Delta^{n-1}}(t) \leq e(t)+\int_{0}^{t_{1}} a(t, s) F(s, x(s)) \Delta s \\
+\int_{t_{1}}^{t} a(t, s) p_{2}(s) x^{\gamma}(s) \Delta s,
\end{gathered}
$$

As in the proof of Theorem 4, we obtain (14) and hence (55) becomes

$$
\begin{aligned}
& \left(r x^{\Delta}\right)^{\Delta^{n-1}}(t) \\
& \leq k+e(t) \\
& \quad+k_{1} \int_{t_{1}}^{t}\left[p_{2}(s) q(s) x^{\gamma}(s)-p(s) x(s)\right] \Delta s \\
& \quad+k_{1} \int_{t_{1}}^{t} p(s) x(s) \Delta s, \quad \text { for } t \geq t_{1} .
\end{aligned}
$$

By applying (6) with

$$
\begin{aligned}
\lambda & =\gamma, \\
X & =\left(p_{2} q\right)^{1 / \gamma} x, \\
Y & =\left(\frac{1}{\gamma} p\left(p_{2} q\right)^{1 / \gamma}\right)^{1 /(\gamma-1)},
\end{aligned}
$$

we have

$$
\begin{gathered}
p_{2}(t) q(t) x^{\gamma}(t)-p(t) x(t) \leq\left((1-\gamma) \gamma^{\gamma /(1-\gamma)}\right) \\
\cdot p^{\gamma /(\gamma-1)}(t) p_{2}^{1 /(1-\gamma)}(t) q^{1 /(1-\gamma)}(t) .
\end{gathered}
$$

Using (58) in (56), we find

$$
\begin{aligned}
& \left(r x^{\Delta}\right)^{\Delta^{n-1}}(t) \leq k+e(t)+k_{1}(1-\beta) \\
& \cdot \beta^{\beta /(\beta-1)} \int_{t_{1}}^{t} p^{\gamma /(\gamma-1)}(s) p_{2}^{1 /(1-\gamma)}(s) q^{1 /(1-\gamma)}(s) \Delta s \\
& \quad+k_{1} \int_{t_{1}}^{t} p(s) x(s) \Delta s, \quad \forall t \geq t_{1} .
\end{aligned}
$$

Integrating this inequality $(n-1)$-times from $t_{1}$ to $t$ and using Lemma 3, we have

$$
\begin{aligned}
& \left(r x^{\Delta}\right)(t) \leq k h_{n-1}\left(t, t_{1}\right)+\sum_{i=0}^{n-2} h_{i}\left(t, t_{1}\right)\left(r x^{\Delta}\right)^{\Delta^{i}}\left(t_{1}\right) \\
& \quad+\int_{t_{1}}^{t} h_{n-2}(t, \sigma(s)) e(s) \Delta s+k_{1} \int_{t_{1}}^{t} h_{n-2}(t, \sigma(u)) \\
& \quad \cdot \int_{t_{1}}^{u} p(s) x(s) \Delta s \Delta u+\left(k_{1}(1-\gamma) \gamma^{\gamma /(1-\gamma)}\right) \\
& \quad \cdot \int_{t_{1}}^{t} h_{n-2}(t, \sigma(u)) \\
& \quad \cdot \int_{t_{1}}^{u} p^{\gamma /(\gamma-1)}(s) p_{2}^{1 /(1-\gamma)}(s) q^{1 /(1-\gamma)}(s) \Delta s \Delta u .
\end{aligned}
$$


As in the proof of Theorem 4, one can easily find

$$
\begin{aligned}
& x^{\Delta}(t) \leq \frac{c}{r(t)} H_{n-1}\left(t, t_{1}\right)+\frac{1}{r(t)} \\
& \cdot \int_{t_{1}}^{t} h_{n-2}(t, \sigma(s)) e(s) \Delta s+k_{1} \frac{t h_{n-2}\left(t, t_{1}\right)}{r(t)} \\
& \cdot \int_{t_{1}}^{t} p(s) x(s) \Delta s+\left(k_{1}(1-\gamma) \gamma^{\gamma /(1-\gamma)}\right) \frac{1}{r(t)} \\
& \cdot \int_{t_{1}}^{t} h_{n-2}(t, \sigma(u)) \\
& \cdot \int_{t_{1}}^{u} p^{\gamma /(\gamma-1)}(s) p_{2}^{1 /(1-\gamma)}(s) q^{1 /(1-\gamma)}(s) \Delta s \Delta u,
\end{aligned}
$$

where $c$ is given by (18). Using condition (50), we see that $t h_{n-2}\left(t, t_{1}\right) / r(t)$ is bounded for $t \geq t_{1}$; say by $k_{3}>0$, and we see that

$$
\begin{aligned}
& x^{\Delta}(t) \leq \frac{c}{r(t)} H_{n-1}\left(t, t_{1}\right)+\frac{1}{r(t)} \\
& \cdot \int_{t_{1}}^{t} h_{n-2}(t, \sigma(s)) e(s) \Delta s+k_{1} k_{3} \int_{t_{1}}^{t} p(s) x(s) \Delta s \\
& +\left(k_{1}(1-\gamma) \gamma^{\gamma /(1-\gamma)}\right) \frac{1}{r(t)} \int_{t_{1}}^{t} h_{n-2}(t, \sigma(u)) \\
& \cdot \int_{t_{1}}^{u} p^{\gamma /(\gamma-1)}(s) p_{2}^{1 /(1-\gamma)}(s) q^{1 /(1-\gamma)}(s) \Delta s \Delta u .
\end{aligned}
$$

Integrating this inequality from $t_{1}$ to $t$ and employing Lemma 3 in [10] to interchange the order of integration we obtain

$$
\begin{aligned}
& x(t) \leq x\left(t_{1}\right)+c H\left(t, t_{1}\right)+\int_{t_{1}}^{t} \frac{1}{r(u)} \\
& \cdot \int_{t_{1}}^{u} h_{n-2}(u, \sigma(s)) e(s) \Delta s \Delta u \\
& +k_{1} k_{3} t \int_{t_{1}}^{t} p(s) x(s) \Delta s+\left(k_{1}(1-\gamma) \gamma^{\gamma /(1-\gamma)}\right) \\
& \cdot \int_{t_{1}}^{t} \frac{1}{r(u)} \int_{t_{1}}^{u} h_{n-2}(t, \sigma(\tau)) \\
& \cdot \int_{t_{1}}^{\tau} p^{\gamma /(\gamma-1)}(s) p_{2}^{1 /(1-\gamma)}(s) q^{1 /(1-\gamma)}(s) \Delta s \Delta \tau \Delta u .
\end{aligned}
$$

Using conditions (49), (50), and (53), there exist positive constants $\bar{c}$ and $\overline{\bar{c}}$ such that

$$
\frac{x(t)}{t} \leq \bar{c}+\overline{\bar{c}} \int_{t_{1}}^{t} s p(s)\left(\frac{x(s)}{s}\right) \Delta s .
$$

Applying Gronwall's inequality [1, Corollary 6.7] to inequality (64) and then using condition (51), we have

$$
\lim _{t \rightarrow \infty} \sup \frac{x(t)}{t}<\infty .
$$

If $x(t)$ is eventually negative, we set $y(t)=-x(t)$ to see that $y(t)$ satisfies (1) with $e(t)$ replaced by $-e(t)$ and $f_{1}(t, x)$ by $f_{1}(t,-y)$.

It follows in a similar manner that

$$
\lim _{t \rightarrow \infty} \sup \frac{-x(t)}{t}<\infty
$$

We conclude from (65) and (66) that (54) holds.

Next by employing Theorem 13 , we present the following oscillation result for (1) with $f_{2}=0$.

Theorem 14. Let conditions (I) and (II) hold with $f_{1}=0$ and $\gamma<1$. Suppose that conditions (50), (52), and (53) hold. If

$$
\begin{aligned}
& \lim _{t \rightarrow \infty} \sup t \int_{t_{0}}^{t} s p(s) \Delta s<\infty, \\
& \lim _{t \rightarrow \infty} \int_{t_{0}}^{t} \frac{1}{r(u)} \int_{t_{0}}^{u} h_{n-2}(u, \sigma(\tau)) \\
& \quad \cdot \int_{t_{0}}^{\tau} p^{\gamma /(\gamma-1)}(s) p_{2}^{1 /(1-\gamma)}(s) q^{1 /(1-\gamma)}(s) \Delta s \Delta \tau \Delta u \\
& <\infty,
\end{aligned}
$$

$$
\begin{aligned}
& \lim _{t \rightarrow \infty} \sup \int_{t_{0}}^{t} \frac{1}{r(u)} \int_{t_{0}}^{u} h_{n-2}(u, \sigma(\tau)) e(s) \Delta s \Delta u \\
& \quad=+\infty \\
& \lim _{t \rightarrow \infty} \inf \int_{t_{0}}^{t} \frac{1}{r(u)} \int_{t_{0}}^{u} h_{n-2}(u, \sigma(s)) e(s) \Delta s \Delta u=-\infty,
\end{aligned}
$$

for all $t_{0} \geq 0$, then (1) is oscillatory.

Proof. Let $x(t)$ be a nonoscillatory solution of (1). First, assume $x(t)$ is eventually positive. Fix $t_{0} \geq 0$ and suppose $x(t)>0$ for $t \geq t_{1}$ for some $t_{1} \geq t_{0}$. The proof when $x(t)$ is eventually negative is similar.

Proceeding as in the proof of Theorem 13, we arrive at (58). Therefore

$$
\begin{gathered}
x(t) \leq x\left(t_{1}\right)+c H\left(t, t_{1}\right)+k_{1} k_{3} t \int_{t_{1}}^{t} s p(s)\left(\frac{x(s)}{s}\right) \Delta s \\
+\int_{t_{1}}^{t} \frac{1}{r(u)} \int_{t_{1}}^{u} h_{n-2}(u, \sigma(s)) e(s) \Delta s \Delta u \\
+\left(k_{1}(1-\gamma) \gamma^{\gamma /(1-\gamma)}\right) \int_{t_{1}}^{t} \frac{1}{r(u)} \int_{t_{1}}^{u} h_{n-2}(t, \sigma(\tau)) \\
. \int_{t_{1}}^{\tau} p^{\gamma /(\gamma-1)}(s) p_{2}^{1 /(1-\gamma)}(s) q^{1 /(1-\gamma)}(s) \Delta s \Delta \tau \Delta u .
\end{gathered}
$$

Conditions (67) and (68) imply conditions (50) and (49), respectively, and so the conclusion of Theorem 13 holds. This together with condition (50) and (68) shows that the second term and last integral are bounded.

Taking lim inf for both sides of (71) as $t \rightarrow \infty$ and using (67) and (70) result in a contradiction with the fact that $x(t)$ is eventually positive. 
Similar to the sublinear case one can easily prove the following theorems for the integrodynamic equation (1) when $\gamma=1$.

Theorem 15. Let conditions (I) and (II) hold with $f_{1}=0$ and $\gamma=1$. Moreover, assume

$$
\int^{\infty} s p_{2}(s) \Delta s<\infty
$$

and conditions (50), (52), and (53) hold for any $t_{0} \geq 0$. Then every nonoscillatory solution of (1) satisfies (54).

Theorem 16. Let conditions (I) and (II) hold with $f_{1}=0$ and $\gamma=1$. In addition, suppose conditions (50), (52), (53), (69), and (70) hold. If

$$
\lim _{t \rightarrow \infty} \sup t \int_{t_{0}}^{t} s p_{2}(s) \Delta s<\infty
$$

for all $t_{0} \geq 0$, then (1) is oscillatory.

Similar to the above results, one can easily prove the following theorems for the integrodynamic equation (1) with $f_{2}=0$.

Theorem 17. Let conditions (I) and (II) hold with $f_{2}=0$ and $\beta>1$. Assume that there exists a rd-continuous function $p$ : $T \rightarrow R^{+}$such that

$$
\begin{aligned}
\lim _{t \rightarrow \infty} \frac{1}{t} \int_{t_{0}}^{t} \frac{1}{r(u)} \int_{t_{0}}^{u} h_{n-2}(u, \sigma(\tau)) \\
\quad \cdot \int_{t_{0}}^{\tau} p^{1 /(1-\beta)}(s) p_{1}^{\beta /(\beta-1)}(s) q^{\beta /(\beta-1)}(s) \Delta s \Delta \tau \Delta u \\
\quad<\infty .
\end{aligned}
$$

If conditions (50)-(53) hold for all $t_{0} \geq 0$, then every nonoscillatory solution of (1) satisfies (54).

Theorem 18. Let conditions (I) and (II) hold with $f_{2}=0$ and $\beta>1$. Assume that there exists a rd-continuous function $p$ : $T \rightarrow R^{+}$such that

$$
\begin{aligned}
& \lim _{t \rightarrow \infty} \int_{t_{0}}^{t} \frac{1}{r(u)} \int_{t_{0}}^{u} h_{n-2}(u, \sigma(\tau)) \\
& \quad \cdot \int_{t_{0}}^{\tau} p^{1 /(1-\beta)}(s) p_{1}^{\beta /(\beta-1)}(s) q^{\beta /(\beta-1)}(s) \Delta s \Delta \tau \Delta u \\
& \quad<\infty, \\
& \lim _{t \rightarrow \infty} \sup t \int_{t_{0}}^{t} s p(s) \Delta s<\infty .
\end{aligned}
$$

If conditions (50), (52), (53), (68), and (69) hold for all $t_{0} \geq 0$, then (1) is oscillatory.

\section{Illustrative Examples}

As we already mentioned, the results of the present paper are new for the cases when $T=R$, that is, the continuous case, or when $T=Z$, that is, the discrete case. As a numerical illustration of our results in Section 3 with $T=R$, we consider the following equation:

$$
\left((1+t)^{3} x^{\prime}(t)\right)^{\prime}+\int_{0}^{t} \frac{x^{\beta}(s)}{\left(1+t^{2}\right)\left(1+s^{2}\right)} d s=t^{p} \sin (t)
$$

with initial conditions $x\left(t_{0}\right)=x_{0}$ and $x^{\prime}\left(t_{0}\right)=x_{0}^{\prime}$. Compare (76) with (1) to get that $r(t)=(1+t)^{3}, n=2, a(t, s)=1 /(1+$ $\left.t^{2}\right)\left(1+s^{2}\right), F(t, s)=x^{\beta}(s)$, and $e(t)=t^{p} \sin (t)$. We can easily show that conditions (I) and (II) are satisfied. Conditions (11) are satisfied only for $p \geq 3$.

Equation (76) can be converted to two simultaneous firstorder ODEs by substituting $(1+t)^{3} x^{\prime}(t)=y$. This will lead to the following system:

$$
\begin{aligned}
& x^{\prime}(t)=\frac{y(t)}{(1+t)^{3}} ; \quad x(0)=x_{0}, \\
& y^{\prime}(t)=t^{p} \sin (t)-\int_{0}^{t} \frac{x^{\beta}(s)}{\left(1+t^{2}\right)\left(1+s^{2}\right)} d s ; \\
& y(0)=x_{0}^{\prime} .
\end{aligned}
$$

Many numerical techniques can be used to solve (77). In the current work, the second-order accurate modified Euler technique is considered. The time interval $\left[t_{0}, T_{f}\right]$ will be divided into $M$ equal subdivisions with $\Delta t$ width for each one. The prediction and correction steps of the modified Euler technique will be

$$
\begin{aligned}
& \bar{x}_{i+1}=x_{i}+\Delta \operatorname{tg}\left(t_{i}, x_{i}, y_{i}\right), \\
& \bar{y}_{i+1}=y_{i}+\Delta \operatorname{th}\left(t_{i}, x_{i}, y_{i}\right), \\
& x_{i+1}=x_{i}+0.5 \Delta t\left[g\left(t_{i}, x_{i}, y_{i}\right)+g\left(t_{i+1}, \bar{x}_{i+1}, \bar{y}_{i+1}\right)\right], \\
& y_{i+1}=y_{i}+0.5 \Delta t\left[h\left(t_{i}, x_{i}, y_{i}\right)+h\left(t_{i+1}, \bar{x}_{i+1}, \bar{y}_{i+1}\right)\right],
\end{aligned}
$$

where

$$
\begin{aligned}
& g(t, x, y)=\frac{y(t)}{(1+t)^{3}}, \\
& h(t, x, y)=t^{p} \sin (t)-\int_{0}^{t} \frac{x^{\beta}(s)}{\left(1+t^{2}\right)\left(1+s^{2}\right)} d s .
\end{aligned}
$$

The integral in (79) can be approximated numerically at each time instant $t_{i}$ using the trapezoidal rule which has accuracy of $O(\Delta t)^{2}$. Solving (76) with $p=1,2,3$, and 4 and $\beta=1 / 3$ with initial conditions $x(0)=0.1$ and $x^{\prime}(0)=0.0$, to get Figures 1 and 2. In Figure $1, p=1,2$, the solution is not oscillatory as conditions (11) are not satisfied. In Figure 2, $p=3,4$, we get oscillatory solution and this example validates numerically Theorem 4 . Similar results are obtained for $\beta=1$ and $\beta=3$.

As another example, consider the following equation:

$$
\left((1+t)^{4} x^{\prime}(t)\right)^{\prime \prime}+\int_{0}^{t} \frac{x^{\gamma}(s)-x^{\beta}(s)}{\left(1+t^{2}\right)\left(1+s^{2}\right)} d s=t^{p} \sin (t)
$$




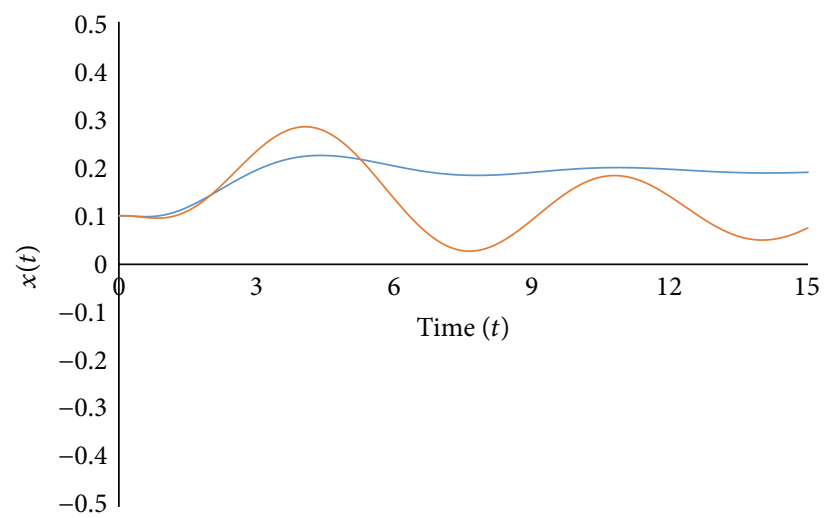

$-p=1$

$-p=2$

FIgURE 1: Solution $x(t)$ of (76) for $\beta=1 / 3$ and $p=1,2$.

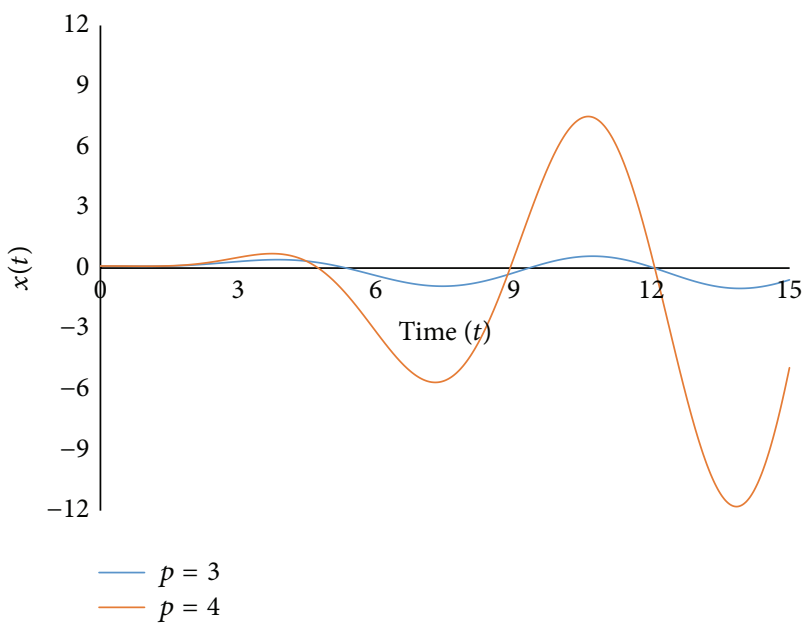

Figure 2: Solution $x(t)$ of (76) for $\beta=1 / 3$ and $p=3,4$.

with initial conditions $x\left(t_{0}\right)=x_{0}, x^{\prime}\left(t_{0}\right)=x_{0}^{\prime}$, and $x^{\prime \prime}\left(t_{0}\right)=$ $x_{0}^{\prime \prime}$. Follow the same procedure above and substitute $(1+$ $t)^{4} x^{\prime}(t)=y$ to get the following system:

$$
\begin{aligned}
& x^{\prime}(t)=\frac{y}{(1+t)^{4}} ; \quad x\left(t_{0}\right)=x_{0}, \\
& y^{\prime}(t)=z ; \quad y\left(t_{0}\right)=\left(1+t_{0}\right)^{4} x^{\prime}\left(t_{0}\right), \\
& z^{\prime}(t)=t^{p} \sin (t)-\int_{0}^{t} \frac{x^{\gamma}(s)-x^{\beta}(s)}{\left(1+t^{2}\right)\left(1+s^{2}\right)} d s ; \\
& z\left(t_{0}\right)=y^{\prime}\left(t_{0}\right)=\left(1+t_{0}\right)^{4} x^{\prime \prime}\left(t_{0}\right)+4\left(1+t_{0}\right)^{3} x^{\prime}\left(t_{0}\right) .
\end{aligned}
$$

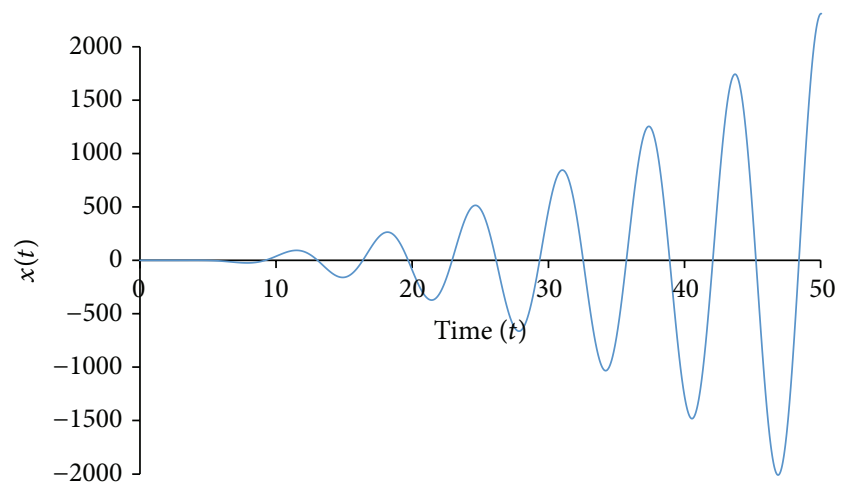

Figure 3: Solution $x(t)$ of (80) for $\gamma=1$ and $\beta=5 / 3$ and $p=6$.

The prediction and correction steps of the modified Euler technique will be

$$
\begin{aligned}
\bar{x}_{i+1} & =x_{i}+\Delta \operatorname{tf}\left(t_{i}, x_{i}, y_{i}, z_{i}\right), \\
\bar{y}_{i+1} & =y_{i}+\Delta \operatorname{tg}\left(t_{i}, x_{i}, y_{i}, z_{i}\right), \\
\bar{z}_{i+1} & =z_{i}+\Delta \operatorname{th}\left(t_{i}, x_{i}, y_{i}, z_{i}\right), \\
x_{i+1} & =x_{i}+0.5 \Delta t\left[f\left(t_{i}, x_{i}, y_{i}, z_{i}\right)\right. \\
+ & \left.f\left(t_{i+1}, \bar{x}_{i+1}, \bar{y}_{i+1}, \bar{z}_{i+1}\right)\right], \\
y_{i+1} & =y_{i}+0.5 \Delta t\left[g\left(t_{i}, x_{i}, y_{i}, z_{i}\right)\right. \\
+ & \left.g\left(t_{i+1}, \bar{x}_{i+1}, \bar{y}_{i+1}, \bar{z}_{i+1}\right)\right], \\
z_{i+1} & =z_{i}+0.5 \Delta t\left[h\left(t_{i}, x_{i}, y_{i}, z_{i}\right)\right. \\
+ & \left.h\left(t_{i+1}, \bar{x}_{i+1}, \bar{y}_{i+1}, \bar{z}_{i+1}\right)\right],
\end{aligned}
$$

where

$$
\begin{aligned}
& f(t, x, y, z)=\frac{y}{(1+t)^{4}}, \\
& g(t, x, y, z)=z \\
& h(t, x, y, z)=t^{p} \sin (t)-\int_{0}^{t} \frac{x^{\gamma}(s)-x^{\beta}(s)}{\left(1+t^{2}\right)\left(1+s^{2}\right)} d s .
\end{aligned}
$$

We will consider the numerical solution of (80) with $p=6$ and with the initial conditions: $x(0)=x^{\prime}(0)=x^{\prime \prime}(0)=0$.

For $\gamma=1$ and $\beta=5 / 3$, the oscillatory solution in Figure 3 is obtained and this validates Theorem 7. Similar results are obtained to validate Theorem 8 (with $\beta=1$ and $\gamma=1 / 3$ ) and Theorems 9 and 10 (with $\beta=5 / 3$ and $\gamma=1 / 3$ ). Theorems $13-18$ can be validated in a similar way.

General Remarks

(1) The results presented in this paper are new for $T=R$ and $T=Z$

(2) The results of this paper are presented in a form which is essentially new for (1) with different nonlinearities. 
(3) The techniques offered in this paper can be employed to Volterra integral equations on timescales of the form

$$
x(t)+\int_{0}^{t} a(t, s) F(s, x(s)) \Delta s=e(t) .
$$

The formulation of our results to the above equation is left to the reader.

(4) The results of this paper can be extended easily to delay integrodynamic equations of the form

$$
\left(r x^{\Delta}\right)^{\Delta^{n-1}}(t)=e(t)-\int_{0}^{t} a(t, s) F(s, x(g(s))) \Delta s
$$

where $g: T \rightarrow T$ is rd-continuous such that $g(t) \leq t$ and $g^{\Delta}(t) \geq 0$ for $t \geq 0$ and $\lim _{t \rightarrow \infty} g(t)=\infty$. The formulation of the results is left to the reader.

(5) We note that we can formulate the obtained results for the timescales $T=R$ (the continuous case), $T=Z$ (the discrete case), $T=q^{N_{0}}$ with $q>1$ (the quantum calculus case), $T=h Z$ with $h>0, T=N_{0}^{2}$, and so forth. The details are left to the reader.

\section{Conflict of Interests}

The authors declare that there is no conflict of interests regarding the publication of this paper.

\section{References}

[1] M. Bohner and A. Peterson, Dynamic Equations on Time-Scales: An Introduction with Applications, Birkhäuser, Boston, Mass, USA, 2001.

[2] G. Karakostas, I. Stavroulakis, and Y. Wu, "Oscillation of Volterra integral equations," Tohoku Mathematical Journal, vol. 45, pp. 583-605, 1993.

[3] B. Karpuz, "Unbounded oscillation of higher-order nonlinear delay dynamic equations of neutral type with oscillating coefficients," Electronic Journal of Qualitative Theory of Differential Equations, no. 34, pp. 1-14, 2009.

[4] H. Onose, "On oscillation of Volterra integral equations and first order functional-differential equations," Hiroshima Mathematical Journal, vol. 20, no. 2, pp. 223-229, 1990.

[5] N. Parhi and N. Misra, "On oscillatory and nonoscillatory behaviour of solutions of Volterra integral equations," Journal of Mathematical Analysis and Applications, vol. 94, no. 1, pp. 137149, 1983.

[6] B. Singh, "On the oscillation of a Volterra integral equation," Czechoslovak Mathematical Journal, vol. 45, no. 4, pp. 699-707, 1995.

[7] S. R. Grace, J. R. Graef, and A. Zafer, "Oscillation of integrodynamic equations on time scales," Applied Mathematics Letters, vol. 26, no. 4, pp. 383-386, 2013.

[8] M. Bohner, S. Grace, and N. Sultana, "Asymptotic behavior of nonoscillatory solutions of higher-order integro-dynamic equations," Opuscula Mathematica, vol. 34, no. 1, pp. 5-14, 2014.

[9] G. Hardy, J. Littlewood, and G. Polya, Inequalities, Cambridge University Press, Cambridge, UK, 1959.
[10] M. Bohner and S. Stevic, "Asymptotic behavior of second order dynamic equations," Applied Mathematics and Computation, vol. 188, pp. 1503-1512, 2007. 


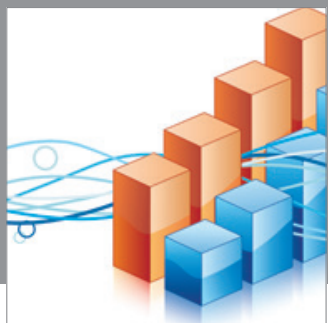

Advances in

Operations Research

mansans

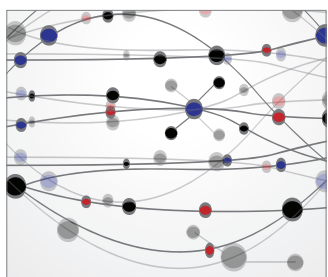

The Scientific World Journal
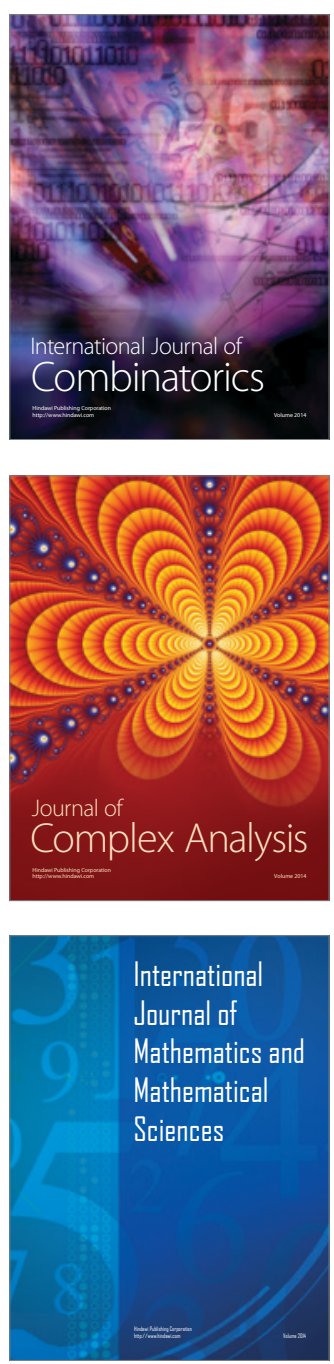
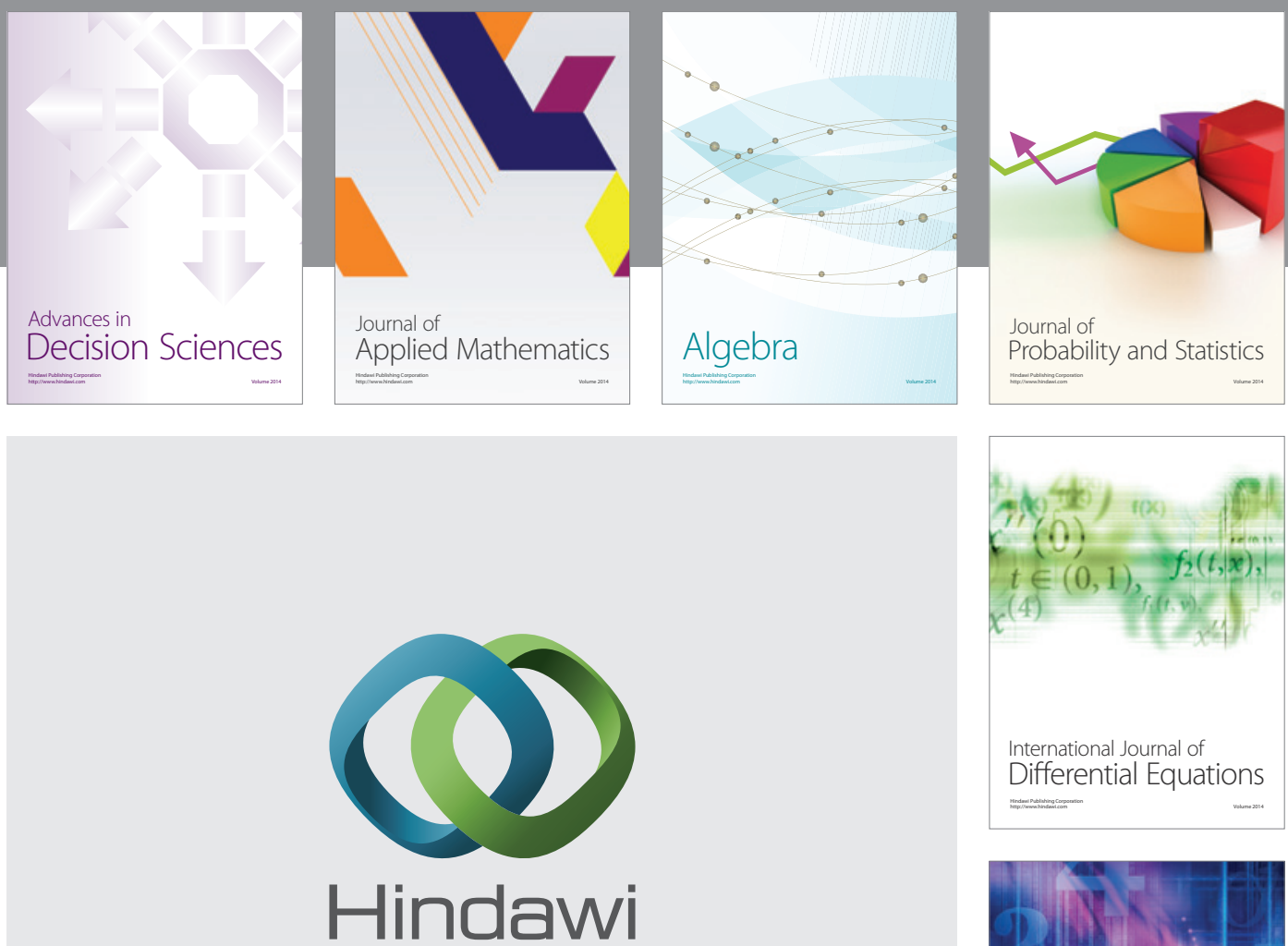

Submit your manuscripts at http://www.hindawi.com
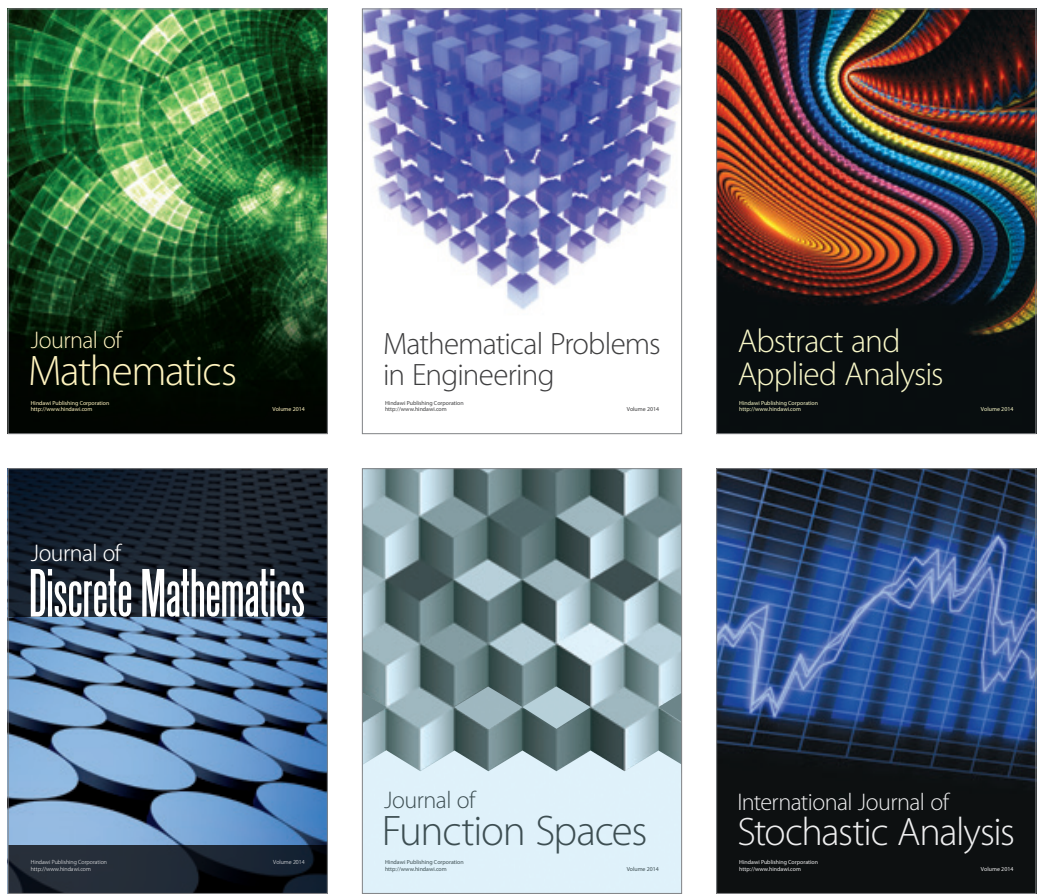

Journal of

Function Spaces

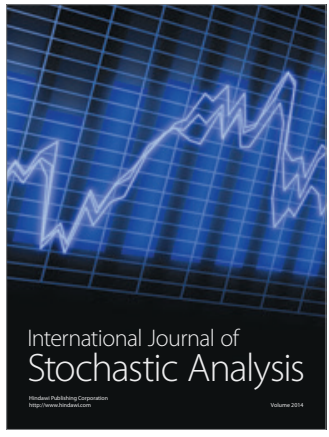

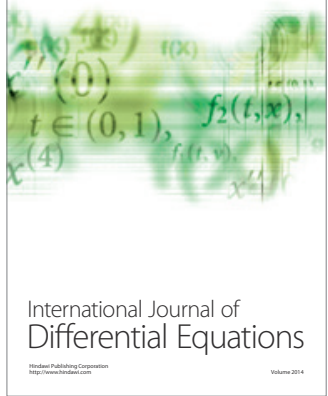
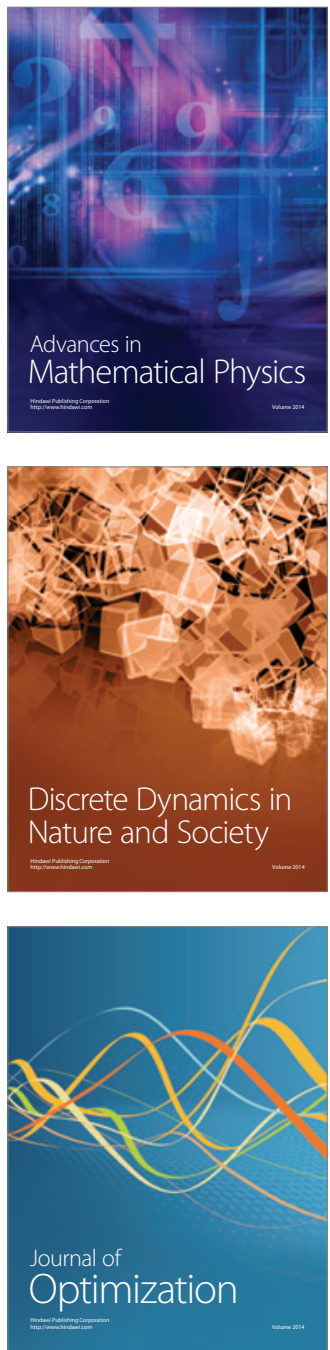